\title{
Independent mitochondrial and nuclear exchanges arising in Rhizophagus irregularis crossed-isolates support the presence of a mitochondrial segregation mechanism
}

\author{
Laurence Daubois $^{\dagger}$, Denis Beaudet ${ }^{\dagger}$, Mohamed Hijri $^{*}$ and Ivan de la Providencia*
}

\begin{abstract}
Background: Arbuscular mycorrhizal fungi (AMF) are members of the phylum Glomeromycota, an early divergent fungal lineage that forms symbiotic associations with the large majority of land plants. These organisms are asexual obligate biotrophs, meaning that they cannot complete their life cycle in the absence of a suitable host. These fungi can exchange genetic information through hyphal fusions (i.e. anastomosis) with genetically compatible isolates belonging to the same species. The occurrence of transient mitochondrial length-heteroplasmy through anastomosis between geographically distant Rhizophagus irregularis isolates was previously demonstrated in single spores resulting from crossing experiments. However, (1) the persistence of this phenomenon in monosporal culture lines from crossed parental isolates, (2) its correlation with nuclear exchanges and (3) the potential mechanisms responsible for mitochondrial inheritance are still unknown. Using the AMF model organism $R$. irregularis, we tested whether the presence of a heteroplasmic state in progeny spores was linked to the occurrence of nuclear exchanges and whether the previously observed heteroplasmic state persisted in monosporal in vitro crossed-culture lines. We also investigated the presence of a putative mitochondrial segregation apparatus in Glomeromycota by identifying proteins similar to those found in other fungal groups.

Results: We observed the occurrence of biparental inheritance both for mitochondrial and nuclear markers tested in single spores obtained from crossed-isolates. However, only one parental mitochondrial DNA and nuclear genotype were recovered in each monosporal crossed-cultures, with an overrepresentation of certain mitochondrial haplotypes. These results strongly support the presence of a nuclear-independent mitochondrial segregation mechanism in $R$. irregularis. Furthermore, a nearly complete set of genes was identified with putative orthology to those found in other fungi and known to be associated with the mitochondrial segregation in Saccharomyces cerevisiae and filamentous fungi.

Conclusions: Our findings suggest that mitochondrial segregation might take place either during spore formation or colony development and that it might be independent of the nuclear segregation machinery. We present the basic building blocks for a better understanding of the mitochondrial inheritance process and segregation in these important symbiotic fungi. The comprehension of these processes is of great importance since it has been shown that different segregated lines of the same isolate can have variable effects on the host plant.
\end{abstract}

Keywords: Arbuscular mycorrhizal fungi, Mitochondrial segregation, Anastomosis, Mitochondrial nucleoids, Protein orthology, Monosporal cultures

\footnotetext{
*Correspondence: mohamed.hijri@umontreal.ca; i.de.la.providencia@

umontreal.ca

${ }^{\dagger}$ Equal contributors

Institut de Recherche en Biologie Végétale, Université de Montréal, 4101

Sherbrooke Est, Montréal H1X 2B2 QC, Canada
}

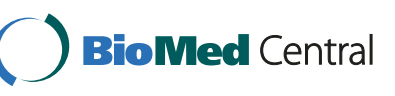

(c) 2016 Daubois et al. Open Access This article is distributed under the terms of the Creative Commons Attribution 4.0 International License (http://creativecommons.org/licenses/by/4.0/), which permits unrestricted use, distribution, and reproduction in any medium, provided you give appropriate credit to the original author(s) and the source, provide a link to the Creative Commons license, and indicate if changes were made. The Creative Commons Public Domain Dedication waiver (http://creativecommons.org/publicdomain/zero/1.0/) applies to the data made available in this article, unless otherwise stated. 


\section{Background}

It has been widely documented that major food crops associate naturally with beneficial soil microbes, including the arbuscular mycorrhizal fungi (AMF), an ancient group of root-inhabiting fungi belonging to the phylum Glomeromycota [1]. These ecologically crucial symbionts enhance the uptake of water and nutrients of plants they colonize, especially phosphate [2], as well as protect them against pathogens [3] and play a key role in soil structure [4]. The benefits to plants provided by this association with AMF could be enhanced by the manipulation of the fungal partner genetics, as demonstrated by Angelard et al. [5] and Colard et al. [6], using the model organism Rhizophagus irregularis. In both studies, through the exchange of nuclear genetic information via hyphal fusion, in vitro crossed-culture lines and segregated culture lines were generated. This process influenced differentially the transcription of symbiosis-specific genes in rice, resulting in an increase in rice growth by a factor of five.

During the last decade numerous studies have been devoted to decipher the nuclear [7] and mitochondrial genome organization patterns $[8,9]$, the sexual/asexual conundrum [10-12] and nuclear segregation process in AMF [13]. These data allowed new insights into the nuclear inheritance processes and segregation mechanisms that could occur in these fungi. Despite this progress, little is known about mitochondrial inheritance in AMF. Since the mitochondrial genome encodes essential components of the cellular energy-producing apparatus, understanding mitochondrial DNA (mtDNA) organization and inheritance in AMF is of paramount importance, in order to manage more efficiently mycorrhizal associations at large scale [14]. A previous study showed that AMF mtDNAs and nuclei migrate massively into spores during their formation [15], but the fate of each parental haplotype following crosses needs to be investigated.

The publication of 14 complete AMF mtDNA [9, 16-20] in the last few years allowed demonstrating that these sequences were all homoplasmic within isolates $[16,17,21,22]$. These studies also showed that genomes are variable in defined intergenic regions between isolates, thus offering an unparalleled opportunity to design isolatespecific markers [8, 17, 20, 23]. Recently, de la Providencia et al. [20] demonstrated length-heteroplasmy in spores formed near anastomosis regions between geographically distant $R$. irregularis in vitro isolates, using isolatespecific mitochondrial markers. However, no information is available regarding the persistence of this length-heteroplasmic state in in vitro monosporal culture lines established from crossed parental isolates. Such a study is needed not only to understand mtDNA inheritance processes and the persistence of mitochondrial haplotypes in stable culture lines, but also to use mtDNA as a criterion to define a reliable AMF taxonomic unit $[8,9]$, either at a genus, species or isolate level.

The mitochondrial inheritance process requires an active transport of organelles along the cytoskeleton and relies on membrane fission and fusion events. It plays a crucial role in the adaptation of the organism in energy requirements. Most of the molecular machinery and cellular mechanisms mediating these processes have been conserved along the fungal and animal kingdom evolution [24]. In filamentous fungi, sexual crosses lead to uniparental transmission of mitochondria $[25,26]$, whereas mitochondria are biparentally inherited in budding yeast $[27,28]$. In yeast, microfilaments, such as actin, play an important role in the positioning and motility of mitochondria, but microtubules are the principal mitochondrial transporter in many other fungi [24]. For many years, the study of mitochondrial dynamics in fungi relied mainly on microscopic observations. However, the sequencing of fungal genomes is an important step towards a better understanding of the molecular machinery mediating mitochondrial inheritance. In that regard, the recently available $R$. irregularis genomic sequence data $[7,29]$ provide new tools to identify molecular components involved in mitochondrial dynamics in AMF.

No study has yet investigated potential mitochondrial segregation and/or inheritance mechanisms occurring in AMF. In the model organism Saccharomyces cerevisiae, mitochondrial inheritance is controlled by the mitochondrial segregation apparatus (MSA), which ensures a reliable transmission of mitochondrial organelles and their genomes to the progeny. Searches of sequence databases reveal evolutionarily conserved proteins for all known budding yeast MSA components in Schizosaccharomyces pombe and in filamentous fungi such as Neurospora crassa and Aspergillus nidulans, notably [30]. This suggests that the core machinery of mitochondrial inheritance has been conserved during evolution, even in organisms that use different cytoskeletal systems for organellar transport [24]. The MSA in S. cerevisiae is a trans-membrane proteic complex consisting of three mitochondrial membrane proteins (i.e. MMM1, MDM10 and MDM12), forming the core component of the apparatus, along with MMM2, MDM31 and MDM32, which interact with the core component (for review see [31]). This proteic complex links the mitochondrial outer membrane to the actin cytoskeleton and the mitochondrial inner membrane to mitochondrial nucleoids, which are clusters of similar mtDNAs packaged by proteins ABF2, ACO1 and ILV5 and maintained together by Holliday junctions [32-34]. However, the molecular machinery of mtDNA inheritance remains largely unknown in many other organisms [35].

In this study, we tested the hypothesis that monosporal crossed-culture lines would maintain the mitochondrial length-heteroplasmy [20] previously observed in $R$. irregularis in vitro spore progenies arising from crossed-cultures 
of genetically divergent isolates. In addition, we investigated nuclear inheritance following anastomosis in spore progenies and monosporal crossed-culture lines. Furthermore, we searched for the existence of a putative MSA in Glomeromycota, by finding the best reciprocal BLAST of yeast MSA proteins in the published genome of $R$. irregularis [7] and its transcriptome [36], and tested their orthology with all available sequences on the database.

\section{Results}

\section{Germination and fungal development of monosporal culture lines}

Cultures from each combination (DAOM-197198/DAOM234328; DAOM-197198/DAOM-240415; DAOM-234328/ DAOM-240415) were performed. After identifying interaction zones between mycelia from different isolates, which were characterized by the formation of hyphal contacts, randomly chosen spores (i.e. progenies) were harvested from the interaction zone of each combination, individually cut out from the mycelium and placed in a new Petri dish in association with Ri T-DNA transformed chicory (Cichorium intybus) roots as described in the methods section (Fig. 1). For each combination,

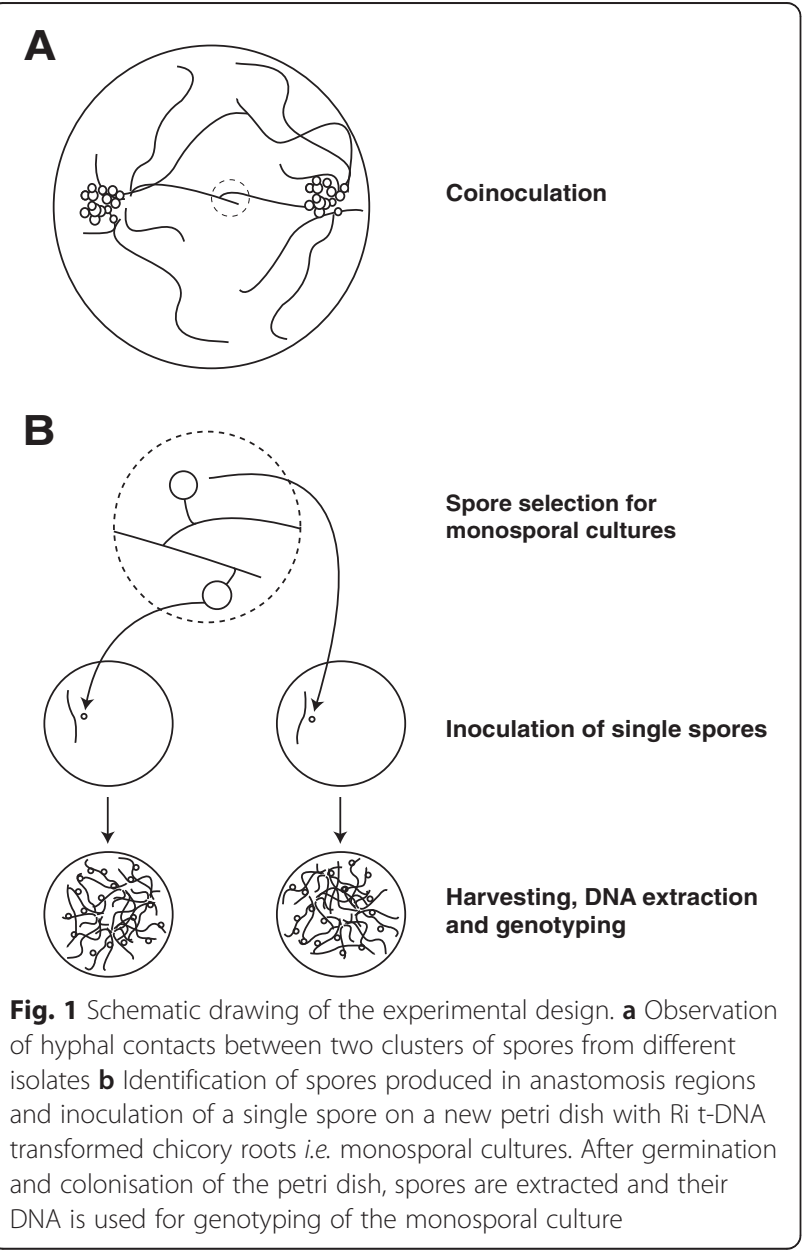

approximately half of the inoculated spores germinated. The observed germination rate corresponds to rates previously observed in four in vitro cultured Glomeraceae species [15]. Following root contact and colonisation, some cultures stopped their growth, without producing any spores, and therefore could not be used in this study. The rate of mycelium development and spore production greatly varied among cultures, the combination of isolates DAOM-197198 and DAOM-240415 being the most successful, resulting in 18 colonized dishes containing between 200 and 1000 newly formed spores. The combination of isolates DAOM-197198 and DAOM-234328 produced seven colonized Petri dishes with few, newly formed spores (10-40 spores/plate). We obtained 11 poorly developed cultures from isolates combination DAOM234328/DAOM-240415, and only nine produced sufficient amount of fungal material required for further analysis. In total, 34 monosporal crossed-cultures lines were genotyped.

\section{Genotyping analysis}

All mitochondrial markers showed to be isolate-specific when challenged against other Rhizophagus isolates (Table 1). The 34 monosporal crossed-culture lines genotyped by qPCR approach presented only one parental mtDNA haplotype. In all nine progenies of the combination DAOM-197198/DAOM-234328, we only detected the DAOM-197198 haplotype, while in the combination DAOM-240415/DAOM-234328, all seven cultures exhibited the DAOM-240415 haplotype. However, among the 18 monosporal cultures of the combination DAOM197198/DAOM-240415, two cultures showed the DAOM197198 haplotype and the other 16 cultures were generated with the DAOM-240415 haplotype. Genotyping results are summarized in Table 2. The qPCR data are shown in the Additional file 1. Interestingly, our results showed an apparent selection and/or segregation bias towards a given haplotype in each combination. Indeed, in combinations where the DAOM-240415 haplotype was present, it seemed to be preferentially selected over the two other haplotypes. Also, the DAOM-197198 haplotype dominated when it was combined with the DAOM234328 haplotype, the latter was not detected in any monosporal culture lines. We also corroborated the heteroplasmy detected in an earlier study by de la Providencia et al. [20], although at a lower rate, using the same genetic material from single spores, thus confirming non-self fusion between genetically-close isolates as an important mechanism shaping genetic exchange (Table 2 and Fig. 2). Two nuclear markers, BG112 and BG62, previously described and suggested for $R$. irregularis genotyping $[5,37,38]$, were used to determine the nuclear inheritance in single spores [20] as well as in 
Table 1 Specificity test of qPCR markers. The marker for each isolate is, at least, specific to the other two isolates used in this study

\begin{tabular}{|c|c|c|c|c|c|c|c|c|c|c|c|}
\hline & \multirow[b]{2}{*}{ Marker\DNA } & \multicolumn{8}{|c|}{ R. irregularis DAOM } & \multirow{2}{*}{$\begin{array}{l}\text { G. cerebriforme } \\
\text { DAOM227022 }\end{array}$} & \multirow[t]{2}{*}{ Wate } \\
\hline & & 197198 & 240415 & 234328 & $197198 / 240415$ & $240415 / 234328$ & $197198 / 234328$ & 242422 & 234179 & & \\
\hline \multirow[t]{3}{*}{ Singleplex } & 197198 & X (19) & - & - & X (19) & - & X (19) & X (19) & - & - & - \\
\hline & 240415 & - & $X(21)$ & - & $X(21)$ & $X(21)$ & - & - & - & - & - \\
\hline & 234328 & - & & $X(26)$ & - & $X(26)$ & $X(27)$ & - & $X(27)$ & - & - \\
\hline \multirow[t]{4}{*}{ Multiplex } & 197198 & X (19) & - & - & X (19) & - & X (19) & - & - & - & - \\
\hline & 240415 & - & $X(21)$ & - & $X(21)$ & $X(21)$ & - & - & - & - & - \\
\hline & 197198 & X (19) & - & - & X (19) & - & X (19) & X (19) & - & - & - \\
\hline & 234328 & - & - & X (26) & - & X (26) & X (27) & - & X (27) & - & - \\
\hline
\end{tabular}

Adequate controls were carried, in triplicate, both in singleplex and multiplex. Multiplex reaction could not be performed with the combination of 240415 and 234328 , because of limitations of the calibrated fluorophores and filter-limitations of the instrument. For each positive assay, in parenthesis is the mean ct value from triplicates with $2 \mathrm{ng}$ of DNA per essay

monosporal crossed-cultures lines. For nuclear markers tested in single spore progenies, we observed different scenarios: (1) only one parental mitochondrial haplotype, with the corresponding parental nuclear genotype (2) one parental mitochondrial haplotype and both parental nuclear genotype (3) both parental mitochondrial haplotypes and only one parental nuclear genotype. Interestingly, out of 12 successfully analysed single spores, four showed the presence of both parental markers. Missing data correspond to incomplete PCR reactions due low amount of genomic DNA remaining from previous experiments published by de la Providencia [20]. In crossed-culture lines, no biparental nuclear inheritance was detected (see Additional file 2).

Mitochondrial segregation machinery and nucleoid genes orthology in $R$. irregularis

The genome of $R$. irregularis DAOM-197198 [7] possesses a nearly full set of genes that exhibited a high degree of similarity to genes coding for the core structure of the MSA and involved in the formation of nucleoids in $S$. cerevisiae and other filamentous fungi (Table 3 and Fig. 3). Orthologous candidates in $R$. irregularis corresponded to the best reciprocal blast hit with the $S$. cerevisiae MSA protein (Table 3). To confirm orthology, phylogenetic trees were constructed using all known orthologous genes in fungi and other organisms. All seven phylogenies performed with clusters of orthologous genes (COGs) supported the idea that $R$. irregularis proteins are putative orthologs of fungal segregation apparatus and nucleoid proteins (see Additional file 3). It is noteworthy that in most cases the $R$. irregularis proteins come out basal in our analyses with low bootstrap support, probably given the lack of sequences availability belonging to lower fungal taxa. The presence of one $S$. cerevisiae paralog was observed in the ACO1 phylogeny, as well as two yeast homologs and one paralog in ABF2 COG phylogeny. However, in both cases, the $R$. irregularis protein grouped with the expected $S$. cerevisiae protein, playing a role in the mitochondrial segregation processes. We did not find any paralogs in $R$. irregularis. Only two genes were missing in $R$. irregularis, i.e. $m d m 31$ and $m d m 32$. However,

Table 2 Characterization of monosporal cultures from crossing experiments with three different Rhizophagus irregularis isolates and single spores from de la Providencia et al. [20]

\begin{tabular}{|c|c|c|c|c|c|c|}
\hline \multirow[t]{2}{*}{ Combinations } & \multirow{2}{*}{$\begin{array}{l}\text { Inoculated monosporal } \\
\text { cultures }\end{array}$} & \multirow{2}{*}{$\begin{array}{l}\text { Colonized monosporal } \\
\text { cultures }\end{array}$} & \multirow{2}{*}{$\begin{array}{l}\text { Spores per } \\
\text { culture }\end{array}$} & \multicolumn{3}{|c|}{ R. irregularis haplotype } \\
\hline & & & & DAOM197198 & DAOM240415 & DAOM234328 \\
\hline R. irregularis 197198/240415 & 50 & 18 & $100-1000$ & 2 & 16 & NA \\
\hline R. irregularis 197198/234328 & 50 & 9 & $50-300$ & 9 & NA & 0 \\
\hline R. irregularis 240415/234328 & 50 & 7 & $30-100$ & NA & 7 & 0 \\
\hline \multicolumn{7}{|c|}{ de la Providencia et al. (2013) [20] } \\
\hline \multirow{2}{*}{ Combinations } & \multicolumn{3}{|l|}{ Heteroplasmic spores } & \multicolumn{3}{|c|}{ Homoplasmic spores } \\
\hline & Previous markers & New markers & & Previous marke & & New markers \\
\hline R. irregularis 197198/240415 & 9 & 3 & & 1 & & 7 \\
\hline R. irregularis 197198/234328 & 5 & 0 & & 5 & & 10 \\
\hline R. irregularis 240415/234328 & 3 & 1 & & 7 & & 9 \\
\hline
\end{tabular}




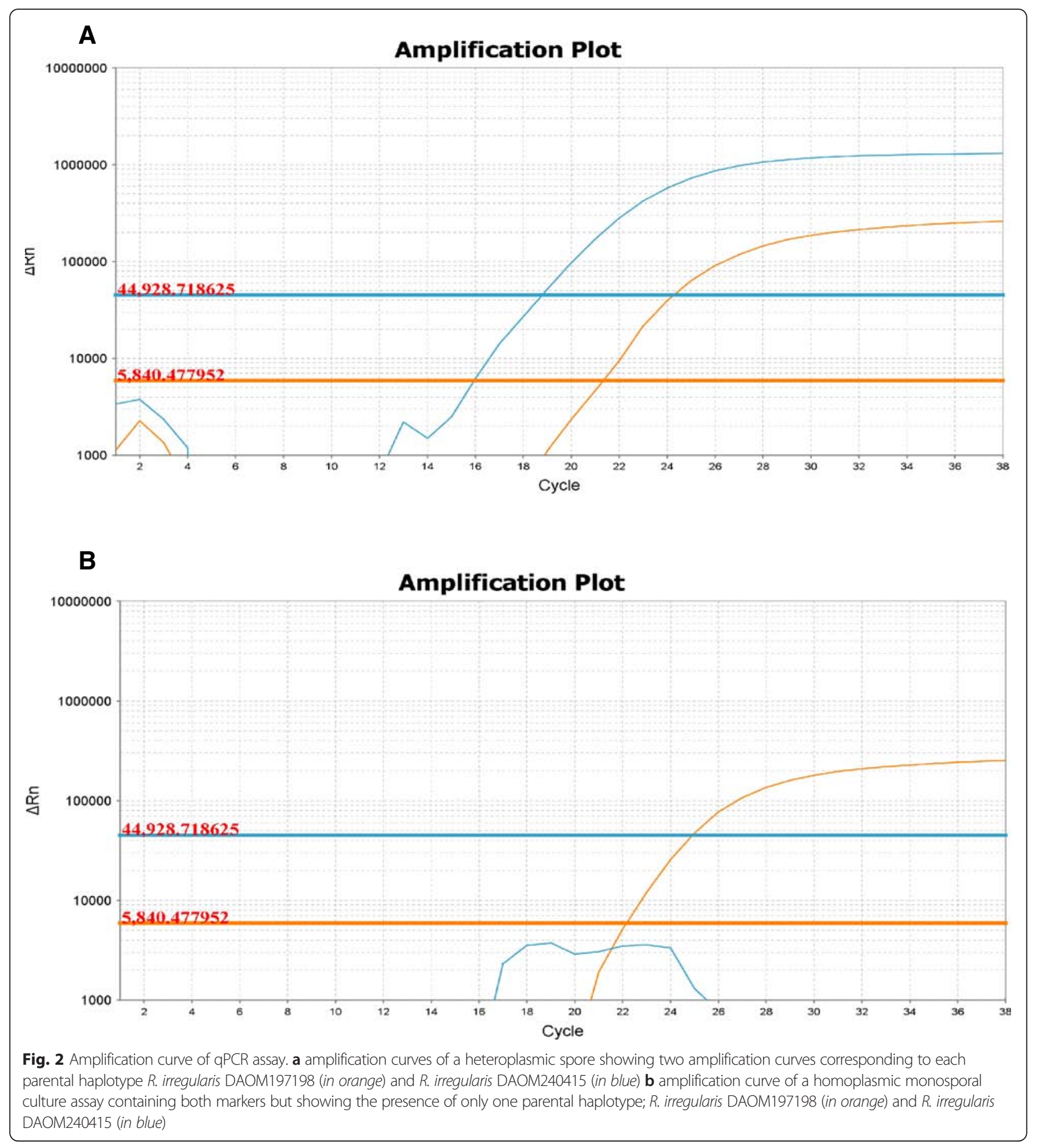

their role in the mitochondrial inheritance process remains unclear in yeast. They are speculated to play an interactive role with the segregation apparatus core components [39]. Furthermore, for these two proteins, the search for COGs recovered only a few yeast sequences, which leads us to believe they might not be necessary to the segregation process in filamentous fungi. All putative orthologs found in $R$. irregularis are likely functional since they are also found in expressed sequence tags (ESTs) [36].

\section{Discussion}

Homoplasmy rather than length-heteroplasmy in monosporal cultures lines from crossed-cultures

Previous results have shown that biparental mtDNA inheritance leading to a heteroplasmic state occurred in 
Table 3 Evidence for Rhizophagus irregularis protein orthology with the Saccharomyces cerevisiae mitochondrial segregation and nucleoid proteic machinery

\begin{tabular}{|c|c|c|c|c|c|c|}
\hline Protein $^{a}$ & Primary function ${ }^{a}$ & Best reciprocal BLAST & E-value & Similarity \% & Phylogenetic orthologs ${ }^{b}$ & mRNA expressionc \\
\hline MDM10 & \multirow{6}{*}{$\begin{array}{l}\text { Core components of the mt segregation } \\
\text { apparatus }\end{array}$} & \multirow[t]{2}{*}{$\bullet$} & \multirow[t]{2}{*}{$1 e-32$} & \multirow[t]{2}{*}{44} & \multirow[t]{2}{*}{$\bullet$} & \multirow[t]{2}{*}{ • } \\
\hline U9UEK3 & & & & & & \\
\hline MDM12 & & \multirow[t]{2}{*}{$\bullet$} & \multirow[t]{2}{*}{$2 \mathrm{e}-15$} & \multirow[t]{2}{*}{52} & \multirow[t]{2}{*}{$\bullet$} & \multirow[t]{2}{*}{$\bullet$} \\
\hline U9UFF5 & & & & & & \\
\hline MMM1 & & \multirow[t]{2}{*}{$\bullet$} & \multirow[t]{2}{*}{$3 e-53$} & \multirow[t]{2}{*}{55} & \multirow[t]{2}{*}{$\bullet$} & \multirow[t]{2}{*}{$\bullet$} \\
\hline U9U1X0 & & & & & & \\
\hline MMM2 & \multirow{4}{*}{$\begin{array}{l}\text { Putative interaction with the mt segregation } \\
\text { apparatus }\end{array}$} & \multirow[t]{2}{*}{$\bullet$} & \multirow[t]{2}{*}{$5 e-11$} & \multirow[t]{2}{*}{53} & \multirow[t]{2}{*}{$\bullet$} & \multirow[t]{2}{*}{$\bullet$} \\
\hline U9TT13 & & & & & & \\
\hline MDM31 & & $\circ$ & $\circ$ & $\circ$ & $\circ$ & $\circ$ \\
\hline MDM32 & & $\circ$ & $\circ$ & $\circ$ & $\circ$ & $\circ$ \\
\hline ILV5 & \multirow[t]{2}{*}{ Biosynthesis of Val, lle and Leu } & \multirow[t]{2}{*}{$\bullet$} & \multirow[t]{2}{*}{$1 e-158$} & \multirow[t]{2}{*}{76} & \multirow[t]{2}{*}{$\bullet$} & \multirow[t]{2}{*}{$\bullet$} \\
\hline U9UJR1 & & & & & & \\
\hline ACO1 & \multirow[t]{2}{*}{ Citric acid cycle } & \multirow[t]{2}{*}{$\bullet$} & \multirow[t]{2}{*}{0.0} & \multirow[t]{2}{*}{84} & \multirow[t]{2}{*}{$\bullet$} & \multirow[t]{2}{*}{$\bullet$} \\
\hline U9U183 & & & & & & \\
\hline $\mathrm{ABF} 2$ & \multirow[t]{2}{*}{ mtDNA packaging } & \multirow[t]{2}{*}{$\pm^{d}$} & \multirow[t]{2}{*}{$1 e-14$} & 48 & $\bullet$ & - \\
\hline U9UF16 & & & & & & \\
\hline
\end{tabular}

${ }^{a}$ Described proteins and known functions in Saccharomyces cerevisiae (Chen et al. [55]), along with the accession number of the $R$. irregularis ortholog gene candidates

${ }^{b}$ As shown in Additional file 3

'Based on the R. irregularis transcriptome data (Tisserant et al. [36])

${ }^{\mathrm{d}}$ Two proteins in $R$. irregularis and S. cerevisiae showed close similarity to each other because they share the same high mobility HMG-BOX proteic domains

the spore progeny from crossed-cultures of divergent $R$. irregularis isolates, however heteroplasmy was not detected in germinated spores [20]. Using TaqMan markers developed in the present study, we did not detect heteroplasmy in monosporal cultures from crossed parental isolates but confirmed the heteroplasmic status of the crossed-cultures spores observed in de la Providencia et al. [20], although at a lower rate. Several factors might explain why we obtained dissimilar results with markers designed in different intergenic regions. The isolatespecific mitochondrial markers are designed in highly variable mobile elements rich regions, which have been shown to be recombination prone [8] and might compromise their specificity. Also, the use of the whole genome amplification (WGA) technique [20] can introduce significant bias regarding the insertions of SNPs or the formation of chimeras [40, 41]. The latter could induce an overestimation of the presence of a given haplotype in a sample, especially since the occurrence of allelic drop-out and preferential amplification is well documented in single cell analysis using a PCR based approach [42]. For these reasons, TaqMan qPCR assay is more reliable to assess mtDNA inheritance, given the nature of the highly variable/dynamic regions in which AMF isolate specific markers are designed.

Earlier studies have demonstrated that genetic exchanges occur via vegetative hyphal fusion (i.e. anastomosis), resulting in nuclei coexisting in a common cytoplasm, altering both the plant and fungal phenotypes $[5,6,13,20,37,43]$. Based on the formation of anastomosis between genetically-close $R$. irregularis isolates, several studies have shown that heterogeneous populations of nuclei $[5,6]$ and mtDNA [20] are randomly inherited at different frequencies (i.e. genetic drift and/ or segregation) [44] into the progeny [15]. These findings support the paradigm that based on the coenocytic nature of the AMF fungal mycelium, nuclei, mitochondria and other organelles can migrate between close or distant regions of genetically-close fungal colonies and form the so-called common mycorrhizal networks (CMN) [45]. However, studies on nuclear dynamics along the symbiotic extraradical mycelium challenged this point of view and brought a somewhat discordant note to the supposed continuous stream of mycelial cytoplasm/protoplasm [46]. Using in vivo two-photon microscopy techniques, these authors revealed a patchy distribution of nuclei throughout the mycelia and also demonstrated that nuclear flow occurs in pulses, being independent from the cytoplasmic streaming. These studies suggest that mitochondria, like nuclei, might not be equally distributed along hyphae. Therefore, through anastomosis originating from different individuals, the inheritance of mitochondria into the spore progeny could be strongly related to the number, type and frequency of the organelle at the 


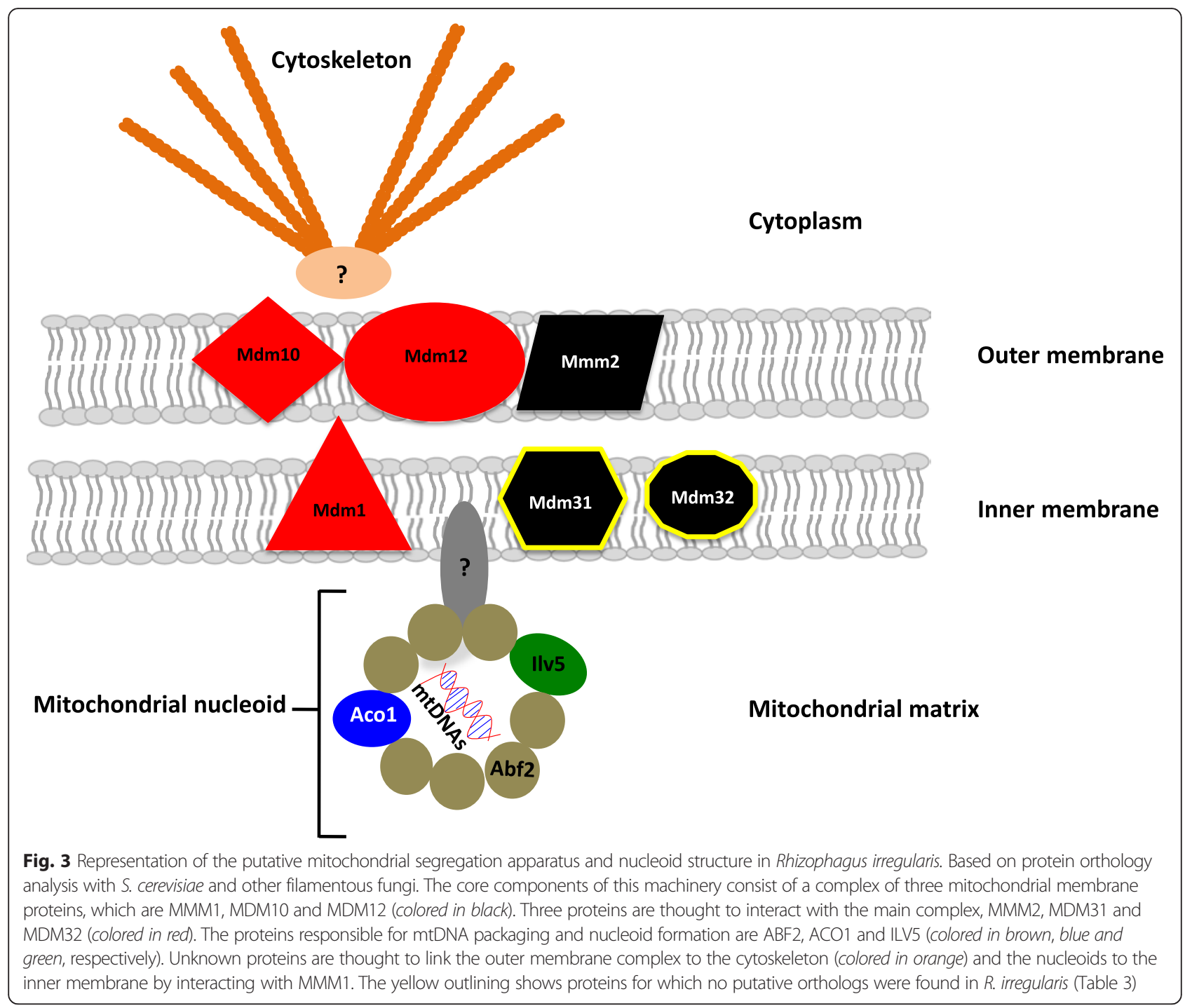

interaction zone (i.e. zone of the mycelium where genetically-close individuals fuse and exchange nutrients and genetic material). The latter could explain the lack of detection of a heteroplasmic state in the progeny spore used to start the monosporal culture. In the event that one haplotype would be crucially underrepresented and therefore the heteroplasmy undetectable by a qPCR assay, such a small amount of a mitochondrial haplotype could easily be lost through stochastic drift in subsequent subcultures, and consequently homoplasmy would persist at the vegetative phase in these fungi. The dominance of some haplotypes we observed would still imply a selection and/or segregation bias of one haplotype over the other.

Together with our observations of a dominant homoplasmic state in the monosporal crossed-culture lines, these studies strongly support a plausible mechanism of mtDNA segregation. This in turn offers an explanation for the low frequency, revised in this study (Table 2), of heteroplasmic spores resulting from crossed, geographically and genetically distant isolates [20] and the nondetection of heteroplasmic monosporal crossed-cultures lines arising from these spores. Moreover, results obtained with nuclear markers show that spores can have both parental nuclear markers, whereas only one mitochondrial parental haplotype. This suggests that both nuclear and mitochondrial inheritance are governed by apparently independent segregation mechanisms.

\section{Evidence for a mitochondrial segregation mechanism in Rhizophagus irregularis}

Although orthology analyses were not entirely conclusive given the basal positioning of the $R$. irregularis sequences in most cases, the identification of putative orthologs in $R$. irregularis genome and transcriptome to the MSA and nucleoid proteins found in other fungi offers a hypothesis to explain the mitochondrial segregation we observed in 
this study. The MMM1-like, MDM10-like and MDM12like proteins are an integral part of the mitochondrial membranes and constitute the core components of the MSA. This proteic complex connects the mitochondrial outer membrane to the cytoskeleton in other fungi [47, 48], while MMM1 is thought to link mitochondrial nucleoids to the inner membrane of the mitochondria [49]. Proteins involved in the MSA have not been reported to play a role in other molecular pathways in S. cerevisiae or filamentous fungi and are well-conserved through fungal evolution [24]. If indeed mitochondria were linked to the cytoskeleton (i.e. either actin or microtubules), along with their mtDNA bound to the inner membrane, their movement through the cytoplasm would not be as free as actually alleged. This hypothesis could potentially explain why the fungal protoplasm moves slower after anastomosis between two divergent isolates as observed earlier [20]. Also, three proteins of the mitochondrial membrane are also proposed to interact with the core components of the MSA, which are MDM31, MDM32 and MMM2. They are thought to play a role in nucleoid stability, mitochondrial morphology and distribution in yeast $[39,50]$. However, we only found a putative ortholog for the MMM2 protein, which might suggest that other proteins could play the same function as MDM31 and MDM32 or that they are not essential in the AMF mitochondrial segregation pathway. Given the phylogenetic divergences between Glomeromycota and higher fungi (i.e. Ascomycota and Basidiomycota) further phylogenetic analysis will need to include putative orthologous and paralogous genes belonging to basal fungi. Currently ongoing sequencing efforts, such as the JGI 1000 fungal genome projects, will greatly help to unravel these evolutionary relationships [51].

Furthermore, we identified putative orthologs for the three proteic constituents of mt-nucleoids in AMF. The ABF2 protein is the core packaging element of $\mathrm{mt}-$ nucleoids in yeast. It is a non-histone DNA binding high mobility group (HMG) protein, which shows homology to nuclear chromatin proteins [52]. This homology explains the difficulty of obtaining the best reciprocal blast (Table 3). The COG used to perform the ABF2 phylogeny (see Additional file 3) included other proteins that were homologs, and also paralogs, since $S$. cerevisiae underwent an ancient genome duplication [53]. The two other essential components of mt-nucleoid are the bifunctional proteins ACO1 and ILV5 $[54,55]$. They are both highly conserved in AMF, probably due to their metabolic role in the citric acid cycle and amino acid biosynthesis, respectively $[31,55,56]$. Their expression is metabolically regulated and they play a role in packaging mtDNA into favourable conformations, while protecting DNA against oxidative stress. The detection in $R$. irregularis genome and transcriptome of well conserved putatively orthologous proteins involved exclusively in the MSA, suggest that they are functional and that they might also be involved in a similar process in AMF. The existence of mt-nucleoids in AMF is important because it accelerates the rate at which mtDNA would segregate, since it is directly correlated to the effective population size [57]. Nucleoids were also shown to create a genetic bottleneck and to be responsible for the rapid mitochondrial segregation observed in yeast [58]. Interspecific differences in the morphology, size and distribution of mitochondrial nucleoids have been visualized using different microscopic methods in yeast $[59,60]$. To further investigate these findings in Glomeromycota, it would be interesting to perform immunofluorescent experiments targeting AMF putative nucleoid proteins, since green fluorescent protein (GFP) tagging has been shown to be transient and render unstable transformants in these multinucleated coenocytic organisms [61].

\section{Conclusions}

Although mitochondrial homoplasmy seems to be the rule rather than the exception in monosporal progeny originating from in vitro crossed-cultures, the coexistence of numerous mtDNA haplotypes in the same cytoplasm and the occurrence of homologous mitochondrial recombination might be common mechanisms in natural populations. Moreover, the observation that mitochondrial and nuclear exchanges are not necessarily correlated suggests the presence of independent mitochondrial and nuclear segregation mechanisms. This supports the presence of a mitochondrial segregation apparatus in $R$. irregularis and provides a better understanding of the mitochondrial inheritance process and segregation in these fungi. Much remains to be learned about how proteins potentially implicated in this segregation mechanism interact with other elements and influence both mtDNA and nuclear inheritance mechanisms in AMF. It would be interesting to study the dynamics of genetic exchanges in natural and/or disturbed environments in order to unravel the underlying segregation processes to better understand (1) further application of mitochondrial markers in population genetics studies and (2) their effects on both the AMF and the host plant fitness.

\section{Methods}

\section{Growth conditions and maintenance of fungal cultures and roots}

Monoxenically produced spores of Rhizophagus irregularis isolates DAOM-197198 (Pont-Rouge, Quebec, Canada), DAOM-234328 (Finland) and DAOM-240415 (Dufrost, Manitoba, Canada) were provided by the DAOM collection (Ottawa, Ontario, Canada). These three isolates were selected because of their different geographical origins and because their mitochondrial genomes have been fully 
sequenced [18, 20]. Spores were subcultured in association with Ri T-DNA transformed chicory (Cichorium intybus) roots on a modified minimal (MM) medium [62] solidified with $0.4 \%(w / v)$ gellan gum (Sigma). Cultures of the three $R$. irregularis isolates were incubated in the dark in an inverted position at $25{ }^{\circ} \mathrm{C}$. Several thousand spores and extraradical mycelia were obtained over a period of 12 weeks. Ri T-DNA transformed chicory roots were routinely propagated by placing actively growing root apexes on MM medium with subsequent incubation at $25{ }^{\circ} \mathrm{C}$ in the dark.

\section{Crossed cultures and monosporal culture lines}

Twenty crossed cultures from each combination (DAOM197198/DAOM-234328; DAOM-197198/DAOM-240415; DAOM-234328/DAOM-240415) were performed by inoculating a hundred spores in close vicinity of Ri T-DNA transformed chicory roots, opposing each other at the extreme side of a Petri plate (Fig. 1). Both colonies were checked weekly and their growth was traced in order to identify interaction zones between mycelia from different isolates, which were characterized by the formation of hyphal contacts. Subsequently, these contacts were checked under a Discovery V12 stereomicroscope (Carl Zeiss, Canada) at magnifications of 6.7-40x. Bright-field microscopy (Axio Imager M1, Carl Zeiss) was also used to observe details of the hyphal interactions at higher magnifications.

After 15 weeks of growth, randomly chosen spores (i.e. progenies) were harvested from the interaction zone of each combination, individually cut out from the mycelium and placed in a new Petri plate $(90 \mathrm{~mm})$ containing MM medium in the close vicinity of a Ri-T transformed chicory root. For each combination, 50 replicates, consisting of one single spore associated with a chicory root were prepared. Each plate was checked weekly for germination, root colonization and colony development over the next 11 weeks.

\section{DNA extraction}

Spores and hyphae were harvested by dissolving the gellan gum matrix in which cultures were grown in a solution containing $0.0083 \mathrm{M}$ sodium citrate and $0.0017 \mathrm{M}$ citric acid. Extracted fungal material was observed under a binocular microscope in order to detect and remove any root contaminants. Spores and hyphae were gently crushed in a $1.5 \mathrm{ml}$ microtube using a sterilized pestle. DNA was extracted using the DNeasy Plant Mini kit (Qiagen, Toronto, $\mathrm{ON})$, according to the manufacturer's instructions.

\section{Mitochondrial marker development, genotyping by real- time PCR and sequencing of progeny spores}

In order to efficiently detect low copy numbers of a given mitochondrial haplotype in each monosporal culture line,
TaqMan isolate-specific markers were developed for each parental isolate and were used to genotype monosporal crossed culture lines (Table 1 and see Additional files 1 and 4). Genotyping of monosporal cultures lines resulting from each crossing experiment were performed in three replicates with approximately $2.5 \mathrm{ng}$ of DNA per replicate. Reactions were carried in $20 \mu \mathrm{l}$, using iTaq $^{\text {Tx }}$ Universal Probes Supermix (Bio-Rad, Canada) with final primers concentration at $0.5 \mu \mathrm{M}$ and final probes concentrations at $0.1 \mu \mathrm{M}$. Reactions were performed using $5^{\prime} \mathrm{FAM}, 5^{\prime} \mathrm{VIC}$ and $5^{\prime} \mathrm{NED}$ dyes and their corresponding quencher. However, due to limited availability of calibrated fluorophores and filter limitations of the instrument, we could only perform multiplex qPCR for combinations DAOM-197198/ DAOM-240415 and DAOM-197198/DAOM-234328 and perform singleplex qPCR reactions for the combination DAOM-240415/DAOM234328. Real time PCR assays were performed on a ViiA ${ }^{\text {tm }} 7$ Real-Time PCR System (LifeTechnologies, Canada). PCR amplicons were visualized on a $2.5 \%$ agarose gel stained with GelRed (Invitrogen, Canada). Successful PCR amplicons were sequenced according to the conventional Sanger technique at the Genome Quebec Innovation Center (Montreal, QC). As a control for marker specificity, the new TaqMan markers developed were challenged against $R$. irregularis (DAOM 242422 and DAOM 234179) and $R$. clarus (MUCL 46238). In addition, these markers were also tested in the spore progeny issued from the previous study of de la Providencia et al. [20], in order to confirm these earlier results.

\section{Sequence-based nuclear markers and genotyping}

In order to assess the nuclear inheritance in single spores and monosporal cultures both arising from crossedisolates, two previously designed and used nuclear markers were tested, locus BG112 [5, 38] and locus BG62 [37] (see Additional file 4). PCR reactions were carried in $25 \mu \mathrm{l}$, using Taq DNA Polymerase (Quiagen, Canada) with final primers concentration at $0.5 \mu \mathrm{M}, \mathrm{dNTP}$ concentration at $0.2 \mathrm{mM}$ and approximately $2.5 \mathrm{ng}$ of DNA. PCR products were separated in a $20 \mathrm{~cm}$ long, $7 \%$ acrylamide gel using DCode Universal Mutation Detection System (Bio-Rad, Canada). Gels were run at $100 \mathrm{vts}, 18 \mathrm{~h}$ for marker BG112 and $15 \mathrm{~h}$ for marker BG62, then stained in a SybrSafe bath for $40 \mathrm{~min}$ and visualized in a Gel Doc XR System (Bio-Rad, Canada) (see Additional file 5).

\section{Protein orthology and phylogenetic analysis}

Each amino acid sequences of the Saccharomyces cerevisiae MSA and/or protein involved in nucleoid formation (i.e. MMM1, MDM10, MDM12, MMM2, MDM31, MDM32, ABF2, ACO1 and ILV5) were searched across the $R$. irregularis DAOM-197198 genome assembly [7] and transcriptome [36], using TBLASTN. Orthologous candidates in $R$. 
irregularis genome were identified using the best-reciprocal BLAST hit to these proteins. Seven putative orthologous sequences were retrieved in $R$. irregularis; Uniprot accession numbers U9U1X0, U9UEK3, U9UFF5, U9TTI3, U9UF16, U9UI83 and U9UJR1, respectively. Furthermore, clusters of orthologous genes (COGs), gathering sequences from numerous organisms, were determined using STRING version 9.05 [63] for each protein candidate. The resulting COGs were aligned using COBALT version 2.01 [64]. Phylogenetic analyses and finding the best evolutionary model was done using the integrative software TOPALI version 2.5 [65]. For each protein candidate, maximum likelihood phylogenetic analyses were done with the closest orthologs found in fungi. Phylogenies were performed accordingly to its predicted model: the MMM1 protein phylogeny was done using the JTT + I + G model, MMM2 with JTT + I + G, MDM12 with JTT + G, MDM10 with WAG + $\mathrm{I}+\mathrm{G}, \mathrm{ACO} 1$ with WAG + I+G, ABF2 with WAG + I+G, and finally the ILV5 phylogeny was performed using the WAG + G model. The robustness of internal branches was evaluated based on 1000 bootstrap replicates $(<60 \%$ cutoff). Tree figures were completed using TreeGraph version 2.0.47 [66].

\section{Availability of supporting data}

$R$. irregularis DAOM-197198 whole genome sequencing project database have been deposited and are available at DNA Data Bank of Japan/European Molecular Biology Laboratory/GenBank (accession no. AUPC0000 0000 or http://www.ncbi.nlm.nih.gov/nuccore/AUPC00 000000). $R$. irregularis DAOM197198 transcriptomic data were retrieved from the INRA GlomusDB website (http://mycor.nancy.inra.fr/IMGC/GlomusGenom e/index3.html). $R$. irregularis seven putative orthologous sequences are available on Uniprot (accession number U9U1X0, U9UEK3, U9UFF5, U9TTI3, U9UF16, U9UI83 and U9UJR1). Proteic sequences are also provided in fasta format in Additional file 6. Other supporting data are presented as additional files. Alignments and phylogenetic trees files are available upon request.

\section{Additional files}

\section{Additional file 1: Table showing qPCR data (ct values) of mitochondrial genotyping. (XLSX $45 \mathrm{~kb}$ )}

Additional file 2: Table showing genotyping of single spores and monosporal cultures arising from crossed isolates. (XLSX $42 \mathrm{~kb}$ )

Additional file 3: Maximum likelihood phylogenetic trees based on seven proteins implicated in the mitochondrial segregation process and nucleoid formation in Saccharomyces cerevisiae, along with their closest orthologs found in fungi and other organisms. Each phylogeny was performed accordingly to its predicted model: the MMM1 protein phylogeny was done using the $J T T+I+G$ model (a), MMM2 with JTT $+I+G(b), M D M 12$ with JTT $+G(c), M D M 10$ with WAG + I+G (d), ACO1 with WAG + I $+G(e), A B F 2$ with WAG $+I+G(f)$, and finally the ILV5 phylogeny was performed using the WAG $+\mathrm{G}$ model (g). Numbers on branches correspond to bootstrap support values (<60\% cut-off) on 1000 replicates. The Ascomycota are in red, the Basidiomycota are in orange, while the Rhizophagus irregularis sequences are in blue. (DOCX $1439 \mathrm{~kb}$ )

Additional file 4: Table showing isolate-specific primers used to discriminate the three Rhizophagus irregularis isolates. (DOCX $76 \mathrm{~kb}$ ) Additional file 5: Polyacrylamid gel electrophoresis (PAGE) on single spores. Lanes $1-2$ and 5-6 represents R. irregularis DAOM197198 and DAOM234328 parental marker BG112 and BG62. Lanes 3-4 and 7-8 shows markers BG112 (3-4) and BG62 (7-8) assays on two single spores containing only the mitochondrial haplotype of $R$. irregularis DAOM197198. (PNG $2768 \mathrm{~kb})$

Additional file 6: $R$. irregularis DAOM197198 sequences. This file contains proteic sequences (fasta format) of seven putative orthologous sequences, also available on Uniprot, accession numbers U9U1X0, U9UEK3, U9UFF5, U9TT13, U9UF16, U9UI83 and U9UJR1. (DOCX 156 kb)

\section{Abbreviations}

ABF: ARS binding factor; ACO: aconitase; AMF: arbuscular mycorrhizal fungi; BLAST: basic local alignment search tool; CMN: common mycorrhizal networks; COGs: cluster of orthologous genes; DAOM: Department of Agriculture, Ottawa, Mycology; DNA: deoxyribonucleic acid; ESTs: expressed sequence tags; HMG: high mobility group; ILV: isoleucine-plus-valine requiring; MDM: maintenance distribution and morphology; MM: modified minimal; MMM: mitochondrial of mitochondrial morphology; MSA: mitochondrial segregation apparatus; mtDNA: mitochondrial DNA; MUCL: Mycothèque de I'Université catholique de Louvain; PAGE: polyacrylamide gel electrophoresis; PCR: polymerase chain reaction; QPCR: quantitative polymerase chain reaction; Ri T-DNA: transferred DNA from the Ri plasmid of Agrobacterium rhizogenes; SNPs: single nucleotide polymorphisms; WGA: whole genome amplification.

\section{Competing interests}

The authors declare that they have no competing interests.

\section{Authors' contributions}

LD carried out the molecular genetic experiments and participated in writing the manuscript. DB performed the gene orthology and phylogenetic analysis and participated in writing the manuscript. $\mathrm{MH}$ participated in the experimental design of the study and revision of the manuscript. IdelaP conceived the overall study and contributed to the writing of the manuscript. All authors approved the final manuscript.

\section{Acknowledgements}

This work was supported by The Natural Sciences and Engineering Research Council of Canada (NSERC) discovery grant to MH (grant number: 328098-2012), which is greatly acknowledged. We would also like to thank Dr. S. Halary for recommendations on the phylogenetic analyses and comments on the manuscript, to David Denis for assistance in the in vitro culture maintenance, set up of the crossed-cultures and the generation and maintenance of the monosporal cultural lines and to Dr. Karen Fisher Favret for comments on the manuscript.

Received: 19 January 2016 Accepted: 20 January 2016 Heln?

\section{References}

1. Schüßler A, Schwarzott D, Walker C. A new fungal phylum, the Glomeromycota: phylogeny and evolution. Mycol Res. 2001:105(12):1413-21.

2. Smith SE, Read DJ. Mycorrhizal symbiosis. London: Academic; 1997.

3. Ismail Y, McCormick S, Hijir M. A fungal symbiont of plant-roots modulates mycotoxin gene expression in the pathogen Fusarium sambucinum. PLoS One. 2011;6(3):e17990.

4. Rillig MC. Arbuscular mycorrhizae, glomalin, and soil aggregation. Can J Soil Sci. 2004;84(4):355-63.

5. Angelard C, Colard A, Niculita-Hirzel H, Croll D, Sanders IR. Segregation in a mycorrhizal fungus alters rice growth and symbiosis-specific gene transcription. Curr Biol. 2010;20(13):1216-21.

6. Colard A, Angelard C, Sanders IR. Genetic exchange in an arbuscular mycorrhizal fungus results in increased rice growth and altered mycorrhizaspecific gene transcription. Appl Environ Microbiol. 2011;77(18):6510-5. 
7. Tisserant E, Malbreil M, Kuo A, Kohler A, Symeonidi A, Balestrini R, et al. Genome of an arbuscular mycorrhizal fungus provides insight into the oldest plant symbiosis. Proc Natl Acad Sci. 2013;110(50):20117-22.

8. Beaudet $D$, Nadimi M, Iffis B, Hijri M. Rapid mitochondrial genome evolution through invasion of mobile elements in two closely related species of arbuscular mycorrhizal fungi. PLoS One. 2013;8(4):e60768.

9. Beaudet D, Terrat $Y$, Halary S, de la Providencia IE, Hijri M. Mitochondrial genome rearrangements in Glomus species triggered by homologous recombination between distinct mtDNA haplotypes. Genome Biol Evol. 2013;5(9):1628-43.

10. Halary S, Daubois L, Terrat Y, Ellenberger S, Wöstemeyer J, Hijri M. Mating type gene homologues and putative sex pheromone-sensing pathway in arbuscular mycorrhizal fungi, a presumably asexual plant root symbiont. PLoS One. 2013;8(11):e80729.

11. Halary S, Malik S-B, Lildhar L, Slamovits CH, Hijri M, Corradi N. Conserved meiotic machinery in glomus spp., a putatively ancient asexual fungal lineage. Genome Biol Evol. 2011;3:950-8.

12. Riley R, Charron P, Idnurm A, Farinelli L, Dalpé $Y$, Martin F, et al. Extreme diversification of the mating type-high-mobility group (MATA-HMG) gene family in a plant-associated arbuscular mycorrhizal fungus. New Phytol. 2014;201(1):254-68.

13. Angelard C, Sanders IR. Effect of segregation and genetic exchange on arbuscular mycorrhizal fungi in colonization of roots. New Phytol. 2011; 189(3):652-7.

14. Ceballos I, Ruiz M, Fernández C, Peña R, Rodríguez A, Sanders IR. The in vitro mass-produced model mycorrhizal fungus, Rhizophagus irregularis, significantly increases yields of the globally important food security crop cassava. PLoS One. 2013;8(8):e70633.

15. Marleau J, Dalpé Y, St-Arnaud M, Hijri M. Spore development and nuclear inheritance in arbuscular mycorrhizal fungi. BMC Evol Biol. 2011;11(1):51.

16. Lee J, Young JPW. The mitochondrial genome sequence of the arbuscular mycorrhizal fungus Glomus intraradices isolate 494 and implications for the phylogenetic placement of Glomus. New Phytol. 2009;183(1):200-11.

17. Formey D, Molès M, Haouy A, Savelli B, Bouchez O, Bécard G, et al. Comparative analysis of mitochondrial genomes of Rhizophagus irregularis-syn. Glomus irregulare-reveals a polymorphism induced by variability generating elements. New Phytol. 2012;196(4):1217-27.

18. Nadimi M, Beaudet D, Forget L, Hijri M, Lang BF. Group I intron-mediated trans-splicing in mitochondria of gigaspora rosea and a robust phylogenetic affiliation of arbuscular mycorrhizal fungi with mortierellales. Mol Biol Evol. 2012;29(9):2199-210.

19. Pelin A, Pombert JF, Salvioli A, Bonen L, Bonfante P, Corradi N. The mitochondrial genome of the arbuscular mycorrhizal fungus Gigaspora margarita reveals two unsuspected trans-splicing events of group I introns. New Phytol. 2012;194(3):836-45.

20. de la Providencia IE, Nadimi M, Beaudet D, Rodriguez Morales G, Hijri M. Detection of a transient mitochondrial DNA heteroplasmy in the progeny of crossed genetically divergent isolates of arbuscular mycorrhizal fungi. New Phytol. 2013;200(1):211-21.

21. Raab PA, Brennwald A, Redecker D. Mitochondrial large ribosomal subunit sequences are homogeneous within isolates of Glomus (arbuscular mycorrhizal fungi, Glomeromycota). Mycol Res. 2005; 109(12):1315-22.

22. Börstler B, Raab PA, Thiéry O, Morton JB, Redecker D. Genetic diversity of the arbuscular mycorrhizal fungus Glomus intraradices as determined by mitochondrial large subunit rRNA gene sequences is considerably higher than previously expected. New Phytol. 2008;180(2):452-65.

23. Corradi N, Bonen L. Mitochondrial genome invaders: an unselfish role as molecular markers. New Phytol. 2012;196(4):963-5.

24. Westermann B, Prokisch $\mathrm{H}$. Mitochondrial dynamics in filamentous fungi. Fungal Genet Biol. 2002;36(2):91-7.

25. Lee SB, Taylor J. Uniparental inheritance and replacement of mitochondrial DNA in Neurospora tetrasperma. Genetics. 1993;134(4):1063-75.

26. Mannella C, Pittenger T, Lambowitz A. Transmission of mitochondrial deoxyribonucleic acid in Neurospora crassa sexual crosses. J Bacteriol. 1979; 137(3):1449.

27. Berger KH, Yaffe MP. Mitochondrial DNA inheritance in Saccharomyces cerevisiae. Trends Microbiol. 2000;8(11):508-13.

28. Okamoto K, Perlman PS, Butow RA. The sorting of mitochondrial DNA and mitochondrial proteins in zygotes: preferential transmission of mitochondrial DNA to the medial bud. J Cell Biol. 1998;142(3):613-23.
29. Lin K, Limpens E, Zhang Z, Ivanov S, Saunders DG, Mu D, et al. Single nucleus genome sequencing reveals high similarity among nuclei of an endomycorrhizal fungus. PLoS Genet. 2014;10(1):e1004078.

30. Boldogh IR, Pon LA. Interactions of mitochondria with the actin cytoskeleton. Biochim Biophys Acta. 2006;1763(5-6):450-62.

31. Chen XJ, Butow RA. The organization and inheritance of the mitochondrial genome. Nat Rev Genet. 2005;6(11):815-25.

32. Lockshon D, Zweifel SG, Freeman-Cook LL, Lorimer HE, Brewer BJ, Fangman WL. A role for recombination junctions in the segregation of mitochondrial DNA in yeast. Cell. 1995;81(6):947-55.

33. White MF, Lilley D. Characterization of a Holliday junction-resolving enzyme from Schizosaccharomyces pombe. Mol Cell Biol. 1997;17(11):6465-71.

34. MacAlpine DM, Perlman PS, Butow RA. The high mobility group protein Abf2p influences the level of yeast mitochondrial DNA recombination intermediates in vivo. Proc Natl Acad Sci. 1998:95(12):6739-43.

35. Birky CW. The inheritance of genes in mitochondria and chloroplasts: laws, mechanisms, and models. Annu Rev Genet. 2001;35(1):125-48.

36. Tisserant E, Kohler A, Dozolme-Seddas P, Balestrini R, Benabdellah K, Colard A, et al. The transcriptome of the arbuscular mycorrhizal fungus Glomus intraradices (DAOM 197198) reveals functional tradeoffs in an obligate symbiont. New Phytol. 2012;193(3):755-69.

37. Croll D, Giovannetti M, Koch AM, Sbrana C, Ehinger M, Lammers PJ, et al. Nonself vegetative fusion and genetic exchange in the arbuscular mycorrhizal fungus Glomus intraradices. New Phytol. 2009;181(4):924-37.

38. Ehinger MO, Croll D, Koch AM, Sanders IR. Significant genetic and phenotypic changes arising from clonal growth of a single spore of an arbuscular mycorrhizal fungus over multiple generations. New Phytologist. 2012;196(3):853-61.

39. Dimmer KS, Jakobs S, Vogel F, Altmann K, Westermann B. Mdm31 and Mdm32 are inner membrane proteins required for maintenance of mitochondrial shape and stability of mitochondrial DNA nucleoids in yeast. J Cell Biol. 2005:168(1):103-15.

40. Pinard R, de Winter A, Sarkis GJ, Gerstein MB, Tartaro KR, Plant RN, et al. Assessment of whole genome amplification-induced bias through high-throughput, massively parallel whole genome sequencing. BMC Genomics. 2006;7(1):216.

41. Lasken RS, Stockwell TB. Mechanism of chimera formation during the multiple displacement amplification reaction. BMC Biotechnol. 2007;7(1):19.

42. Findlay I, Urquhart A, Quirke P, Sullivan K, Rutherford AJ, Litford RJ. Simultaneous DNA fingerprinting', diagnosis of sex and single-gene defect status from single cells. Mol Hum Reprod. 1995;1(2):85-93.

43. Giovannetti M, Azzolini D, Citernesi AS: Anastomosis formation and nuclear and protoplasmic exchange in arbuscular mycorrhizal fungi. Applied and Environmental Microbiology. 1999;65(12):5571-5.

44. Boon E, Zimmerman E, St-Arnaud M, Hijri M. Allelic differences within and among sister spores of the arbuscular mycorrhizal fungus glomus etunicatum suggest segregation at sporulation. PLoS One. 2013;8(12): e83301.

45. Selosse M-A, Richard F, He X, Simard SW. Mycorrhizal networks: des liaisons dangereuses? Trends Ecol Evol (Personal edition). 2006;21(11):621-8.

46. Bago B, Zipfel W, Williams R, Piché Y. Nuclei of symbiotic arbuscular mycorrhizal fungi as revealed by in vivo two-photon microscopy. Protoplasma. 1999;209(1-2):77-89.

47. Boldogh I, Vojtov N, Karmon S, Pon LA. Interaction between mitochondria and the actin cytoskeleton in budding yeast requires two integral mitochondrial outer membrane proteins, Mmm1p and Mdm10p. J Cell Biol. 1998;141(6):1371-81.

48. Boldogh IR, Nowakowski DW, Yang H-C, Chung H, Karmon S, Royes P, et al. A protein complex containing Mdm10p, Mdm12p, and Mmm1p links mitochondrial membranes and DNA to the cytoskeleton-based segregation machinery. Mol Biol Cell. 2003;14(11):4618-27.

49. Hobbs AEA, Srinivasan M, McCaffery JM, Jensen RE. Mmm1p, a mitochondrial outer membrane protein, is connected to mitochondrial DNA (mtDNA) nucleoids and required for mtDNA stability. J Cell Biol. 2001;152(2): 401-10.

50. Youngman MJ, Hobbs AEA, Burgess SM, Srinivasan M, Jensen RE. Mmm2p, a mitochondrial outer membrane protein required for yeast mitochondrial shape and maintenance of mtDNA nucleoids. J Cell Biol. 2004;164(5):677-88.

51. Grigoriev IV, Nordberg H, Shabalov I, Aerts A, Cantor M, Goodstein D, et al. The genome portal of the department of energy joint genome institute. Nucleic Acids Res. 2011;40(Database issue):D26-32. doi:10.1093/nar/gkr947. 
52. Landsman D, Bustin M. A signature for the HMG-1 box DNA-binding proteins. Bioessays. 1993;15(8):539-46.

53. Kellis M, Birren BW, Lander ES. Proof and evolutionary analysis of ancient genome duplication in the yeast Saccharomyces cerevisiae. Nature. 2004; 428(6983):617-24.

54. Bateman JM, Perlman PS, Butow RA. Mutational bisection of the mitochondrial DNA stability and amino acid biosynthetic functions of ilv5 $p$ of budding yeast. Genetics. 2002;161(3):1043-52.

55. Chen XJ, Wang X, Kaufman BA, Butow RA. Aconitase couples metabolic regulation to mitochondrial DNA maintenance. Science. 2005;307(5710):714-7.

56. Petersen JGL, Holmberg S. The ILVS gene of Saccharomyces cerevisiae is highly expressed. Nucleic Acids Res. 1986;14(24):9631-51.

57. White DJ, Wolff JN, Pierson M, Gemmell NJ. Revealing the hidden complexities of mtDNA inheritance. Mol Ecol. 2008;17(23):4925-42.

58. Birky Jr CW, Strausberg RL, Forster JL, Perlman PS. Vegetative segregation of mitochondria in yeast: estimating parameters using a random model. Mol Gen Genet. 1978;158(3):251-61.

59. Williamson D, Fennell D. The use of fluorescent DNA-binding agent for detecting and separating yeast mitochondrial DNA. Methods Cell Biol. 1976;12:335-51.

60. Miyakawa I, Sando N, Kawano S, Nakamura SI, Kuroiwa T. Isolation of morphologically intact mitochondrial nucleoids from the yeast, Saccharomyces cerevisiae. J Cell Sci. 1987;88(4):431-9.

61. Helber N, Requena N. Expression of the fluorescence markers DsRed and GFP fused to a nuclear localization signal in the arbuscular mycorrhizal fungus Glomus intraradices. New Phytol. 2008;177(2):537-48.

62. Bécard G, Fortin JA. Early events of vesicular-arbuscular mycorrhiza formation on Ri T-DNA transformed roots. New Phytol. 1988;108(2):211-8.

63. Von Mering C, Jensen LJ, Snel B, Hooper SD, Krupp M, Foglierini M, et al. STRING: known and predicted protein-protein associations, integrated and transferred across organisms. Nucleic Acids Res. 2005;33 suppl 1:D433-7.

64. Papadopoulos JS, Agarwala R. COBALT: constraint-based alignment tool for multiple protein sequences. Bioinformatics. 2007;23(9):1073-9.

65. Milne I, Wright F, Rowe G, Marshall DF, Husmeier D, McGuire G. TOPALi: software for automatic identification of recombinant sequences within DNA multiple alignments. Bioinformatics. 2004;20(11):1806-7.

66. Stöver BC, Müller KF. TreeGraph 2: combining and visualizing evidence from different phylogenetic analyses. BMC Bioinformatics. 2010;11(1):7.

\section{Submit your next manuscript to BioMed Central and we will help you at every step:}

- We accept pre-submission inquiries

- Our selector tool helps you to find the most relevant journal

- We provide round the clock customer support

- Convenient online submission

- Thorough peer review

- Inclusion in PubMed and all major indexing services

- Maximum visibility for your research

Submit your manuscript at www.biomedcentral.com/submit 